\title{
Modelling In Vivo HIV Dynamics under Combined Antiretroviral Treatment
}

\author{
B. Mobisa ${ }^{1}{ }^{1}{ }^{1}$ G. O. Lawi, ${ }^{2}$ and J. K. Nthiiri ${ }^{2}$ \\ ${ }^{1}$ Department of Mathematics, Laikipia University, P.O. Box 1100-20300, Nyahururu, Kenya \\ ${ }^{2}$ Department of Mathematics, Masinde Muliro University of Science and Technology, P.O. Box 190-50100, Kakamega, Kenya
}

Correspondence should be addressed to B. Mobisa; bmobisa@laikipia.ac.ke

Received 19 September 2018; Revised 19 November 2018; Accepted 26 November 2018; Published 10 December 2018

Academic Editor: Qingdu Li

Copyright (C) 2018 B. Mobisa et al. This is an open access article distributed under the Creative Commons Attribution License, which permits unrestricted use, distribution, and reproduction in any medium, provided the original work is properly cited.

In this paper a within host mathematical model for Human Immunodeficiency Virus (HIV) transmission incorporating treatment is formulated. The model takes into account the efficacy of combined antiretroviral treatment on viral growth and T cell population in the human blood. The existence of an infection free and positive endemic equilibrium is established. The basic reproduction number $R_{0}$ is derived using the method of next generation matrix. We perform local and global stability analysis of the equilibria points and show that if $R_{0}<1$, then the infection free equilibrium is globally asymptotically stable and theoretically the virus is cleared and the disease dies out and if $R_{0}>1$, then the endemic equilibrium is globally asymptotically stable implying that the virus persists within the host. Numerical simulations are carried out to investigate the effect of treatment on the within host infection dynamics.

\section{Introduction}

Human Immunodeficiency Virus (HIV) remains a major threat to human life for the last three and half decades. HIV infection in humans causes Acquired Immunodeficiency Virus (AIDS), a disease that has ravaged human population all over the world. Since its discovery in the early 1980s, there has been tremendous research work on how to contain or eradicate the disease. Mathematical modelling of viral infections has led to greater understanding of virus dynamics and helped in predicting and controlling the spread of viral diseases such as HIV, Hepatitis B Virus (HBV), Hepatitis C Virus (HCV), and Dengue Fever. One of the early models of HIV infection known as the basic model was used by Nowak and May [1] and by Perelson and Nelson (1999) and was successful in numerically reproducing the dynamics of the early stages of HIV and its target CD4+ cells following an infection event. Recent studies have focused on HIV viral and cellular infections incorporating dynamics such as intracellular delays, latent infection and viral mutation, and spatial heterogeneity [2-5]. For instance, [6] investigated the global stability of within host virus models with cell-to-cell viral transmission and obtained a complete analytic description of equilibria. A four-dimensional system of delayed differential equations, where the production and removal rates of the virus and cells are given by general nonlinear functions, was proposed by [4]. Their model investigated the dynamical behaviour of virus target and cell target incidences incorporating humoral immune response. They established three key equilibrium results, an infection free equilibrium, a chronic free equilibrium with inactive humoral immune response, and chronic infection equilibrium with active humoral immune response. With dynamics governed by two bifurcation parameters basic reproduction numbers $\widetilde{R}_{0}^{c}$ and the humoral immunity numbers $\widetilde{R}_{1}^{G}$ and using Lyapunov functionals and Lasalle's invariance principle, the authors proved the global stability of the equilibria.

The inclusion of treatment, at within and between host levels in mathematical modelling, has gained considerable attention in recent years. For instance, at between host levels, epidemiological models with saturated treatment function have been proposed by $[7,8]$. Research on within host models that incorporate treatment has been carried out over the years, with early models highlighting the effects of AZT on viral replication [9]. Among the key findings, viral decline is drug dependent. A study of combined drug therapy of HIV infection was conducted by [10]; the mathematical model developed was used to simulate chemotherapy treatment of HIV infection. The simulations were based on clinical data 
of treatment with combinations of antiviral drugs involving reverse transcriptase inhibitors (RTI) and protease inhibitors (PI) and focused on the timing of treatment. The findings revealed that the success of treatment is based on longer survival times equated to the CD4+ T cells. Global dynamics of delay distributed HIV infection models with differential drug efficacy in cocirculating target cells was investigated [3]. Recent work by [11] sought to mathematically analyze the potential of Prophylaxis treatment in preventing and slowing the spread of HIV/ AIDS in the population. In this study early use of Prophylaxis drug was shown to slow the rate of HIV transmission.

Whereas extensive research on HIV transmission dynamics has been carried out, mathematical modelling of HIV with combined treatment still remains an area of active research among mathematicians and biologists.

In this paper, we propose a within host HIV infection model with a logistic incidence rate that explicitly incorporates the two levels of antiretroviral treatment, namely, the reverse transcriptase inhibitors (RTIs) which prevent the reverse transcription of viral RNA into DNA. In this way the RTIs serve to reduce the rate of infection of activated $C D 4^{+} T$ cells. The other category is protease inhibitors (PIs) which prevent HIV-1 protease from clearing the HIV polyprotein into functional units, thereby causing infected cells to produce immature virus particles that are not capable of infecting additional cells; hence PIs decrease the number of newly infectious virus (virions) that are produced [12]. A mathematical analysis of the effects of treatment on the within host infection dynamics is carried out.

\section{Model Description and Formulation}

A mathematical model of within host HIV infection dynamics is considered. The model is composed of three interacting variables, namely, uninfected CD4+ T cells $T(t)$, actively infected cells $T^{\star}(t)$, and free virus particles $V(t)$. The uninfected CD4+ T cells are produced at rate $r$ and die naturally at the rate $\mu$. The total number of T cells in the body remains bounded; thus the growth of T cells is governed by the logistic proliferation term $\left(1-T / T_{\max }\right)$ which limits $T$ cell growth as the cell population approaches the $\operatorname{limit} T_{\max }$. The uninfected CD4+ $\mathrm{T}$ cells become infected by free virus and actively infected cells according to the simple mass infection terms $\beta V T$ and $\alpha T T^{\star}$, respectively. This generates actively infected cells, $T^{\star}$, which die naturally at the rate $\kappa$. The infected cells produce free viruses $V$ at the rate $\omega \kappa T^{\star}$ and are cleared from circulation at rate $c$ per virus. This viral decline is a function of the efficiency of the combined treatment of Reverse Transcriptase Inhibitor (RTI) and Protease Inhibitor (PI), which are represented by the parameters $\rho$ and $\vartheta$, respectively. From the description and definitions made, the infection dynamics are summarized by the following system of ODEs:

$$
\begin{aligned}
\frac{d T}{d t}= & r T\left(1-\frac{T}{T_{\text {max }}}\right)-(1-\rho) \beta V T-(1-\vartheta) \alpha T T^{\star} \\
& -\mu T \\
\frac{d T^{\star}}{d t}= & (1-\rho) \beta V T+(1-\vartheta) \alpha T T^{\star}-\kappa T^{\star} \\
\frac{d V}{d t}= & \omega \kappa T^{\star}-c V
\end{aligned}
$$

\section{Analysis of the Model}

Since the model describes cell and virus populations dynamics, all the model variables are nonnegative for $t \geq 0$. In the absence of the virus, the $\mathrm{T}$ cell population has a steady state value $T_{0}$; hence the initial conditions for the model (1) are $T(0)=T_{0}>0, T^{\star}(0)=T_{0}^{\star} \geq 0$, and $V(0)=V_{0}>0$. It can be shown that with positive initial data the solutions of model (1) will remain positive and bounded in the feasible region $\Omega=\left\{\left(T, T^{\star}, V\right) \in \mathbb{R}_{+}^{3}: T+T^{\star}+V \leq T_{\text {max }}(1-\mu / r)\right\}$, $\forall t$.

3.1. Basic Reproduction Number. The basic reproduction number $R_{0}$ is defined as the average number of secondary infections produced by one infectious virion and one infected cell over the course of their infectious period in uninfected CD4+ T cell population. We compute $R_{0}$ for model (1) using the next generation matrix method as used in $[13,14]$. Model (1) has two infected compartments $T^{\star}$ and $V$. Let $f_{i}$ be the rate of appearance of new infections in compartment $i$ and $v_{i}$ as the transfer of individuals out of compartment $i$ for the two compartments, respectively, and are given in partitioned form as follows:

$$
f=\left(\begin{array}{c}
(1-\rho) \beta V T+\alpha(1-\vartheta) T T^{\star} \\
0
\end{array}\right)
$$

and

$$
v=\left(\begin{array}{c}
\kappa T^{\star} \\
-\omega \kappa T^{\star}+c V
\end{array}\right)
$$

The Jacobian of $f$ and $v$ evaluated at the Infection Free Equilibrium $E_{0}=\left(T_{\max }, 0,0\right)$ yields

$$
\begin{aligned}
F & =\left(\begin{array}{cc}
\alpha(1-\vartheta) T_{0} & \beta(1-\rho) T_{0} \\
0 & 0
\end{array}\right) \\
V & =\left(\begin{array}{cc}
\kappa & 0 \\
-\omega \kappa & c
\end{array}\right)
\end{aligned}
$$

where $F$ is nonnegative and $V$ is nonsingular. The basic reproduction number is thus given by $R_{0}=\rho\left(F V^{-1}\right)$, where $\rho\left(F V^{-1}\right)$ is the spectral radius of the matrix $F V^{-1}$. Hence

$$
\begin{aligned}
R_{0}= & T_{\max }\left(1-\frac{\mu}{r}\right)(1-\vartheta) \frac{\alpha}{\kappa} \\
& +T_{\max }\left(1-\frac{\mu}{r}\right) \frac{\omega \beta}{c}(1-\rho)
\end{aligned}
$$

3.2. Local Stability Analysis of the Infection Free Equilibrium. We investigate the local stability properties of the infection free equilibrium by approximating the nonlinear system of the differential equations (1) with the linear system at the infection free equilibrium $E_{0}=\left(T_{\max }(1-\mu / r), 0,0\right)$.

Theorem 1. The infection free equilibrium $E_{0}$ is locally asymptotically stable if and only if $R_{0}<1$. 
Proof. Evaluating the Jacobian of model (1) at $E_{0}$, we obtain

$$
J\left(E_{0}\right)=\left(\begin{array}{ccc}
\mu-r & -\alpha T_{\max }\left(1-\frac{\mu}{r}\right)(1-\vartheta) & -\beta T_{\max }\left(1-\frac{\mu}{r}\right)(1-\rho) \\
0 & \alpha T_{\max }\left(1-\frac{\mu}{r}\right)(1-\vartheta)-\kappa & \beta T_{\max }\left(1-\frac{\mu}{r}\right)(1-\rho) \\
0 & \omega \kappa & -c
\end{array}\right)
$$

and clearly

$$
\lambda_{1}=\mu-r
$$

is one of the eigenvalues of the matrix in (7), which is negative because for a population that is growing in numbers; the rate of production (birth rate) is greater than the death rate, that is $r>\mu$. The nature of the remaining roots of (7) can be determined from the reduced matrix:

A

$$
=\left(\begin{array}{cc}
\alpha T_{\max }\left(1-\frac{\mu}{r}\right)(1-\vartheta)-\kappa & \beta T_{\max }\left(1-\frac{\mu}{r}\right)(1-\rho) \\
\omega \kappa & -c
\end{array}\right)
$$

Using Routh-Hurwitz stability criteria, matrix $A$ in (9) will have negative real roots if and only if the $\operatorname{tr} A<0$ and $\operatorname{det} A>$ 0 ; thus

$$
\operatorname{tr} A=R_{0}-1-T_{\max }\left(1-\frac{\mu}{r}\right)(1-\rho) \frac{\beta \omega}{c}-\frac{c}{k}
$$

and

$$
\begin{array}{r}
\operatorname{det} A=-\left[\frac{\alpha}{\kappa} T_{\max }\left(1-\frac{\mu}{r}\right)(1-\vartheta)\right. \\
\left.+\frac{\beta \omega}{c} T_{\max }\left(1-\frac{\mu}{r}\right)(1-\rho)\right]+1
\end{array}
$$

and using (6), (11) reduces to

$$
\operatorname{det} A=1-R_{0}
$$

From (10) and (12), $\operatorname{tr} A<0$ and $\operatorname{det} A>0$ if and only if $R_{0}<$ 1 . Thus $E_{0}$ is locally asymptotically stable whenever $R_{0}<1$ and unstable otherwise.

This means that if a small number of free virus particles enter the blood stream, each virus will infect on average less than one uninfected cell in its entire period of infectivity whenever $R_{0}<1$. Theoretically this shows that the virus is cleared from the body if $R_{0}<1$.

3.3. Global Stability Analysis of the Infection Free Equilibrium. In this section we study the global stability of the infection free equilibrium of model (1) using the theorem by CastilloChavez et al. [13]. We rewrite model (1) in the form

$$
\begin{aligned}
\frac{d X}{d t} & =H(X, Z) \\
\frac{d Z}{d t} & =G(X, Z), \\
G(X, 0) & =0
\end{aligned}
$$

where $X \in \mathbb{R}$ denotes the number of uninfected cells and $Z \in$ $\mathbb{R}^{2}$ denotes the number of actively infected cells and free virus particles, respectively. The infection free equilibrium (IFE) is now denoted by

$$
U_{0}=\left(X^{0}, 0\right), \quad X^{0}=T_{\max }\left(1-\frac{\mu}{r}\right)
$$

The conditions $H_{1}$ and $H_{2}$ below must be met in order to guarantee global asymptotic stability:

(i) $\left(H_{1}\right)$ For $d X / d t=H(X, 0), X^{0}$ is Globally Asymptotically Stable (GAS)

(ii) $\left(H_{2}\right) G(X, Z)=P Z-\widehat{G}(X, Z), \widehat{G}(X, Z) \geq 0$, for $(X, Z) \in \Omega$

where $P=D_{Z} G\left(X^{0}, 0\right)$ is an M-matrix (the off-diagonal elements of $P$ are nonnegative) and $\Omega$ is the region where the model makes biological sense. If system (13) satisfies conditions $\mathrm{H}_{1}$ and $\mathrm{H}_{2}$, then the following theorem holds.

Theorem 2. The fixed point $U_{0}=\left(X^{0}, 0\right)$ is Globally Asymptotically Stable equilibrium of (13) provided that $R_{0}<1$ and that assumptions $\left(\mathrm{H}_{1}\right)$ and $\left(\mathrm{H}_{2}\right)$ are satisfied.

Proof. Let $X(t)=T(t), Z=\left(T^{\star}(t), V(t)\right), H(X, 0)=$ $\left(\begin{array}{c}r T\left(1-T / T_{\max }\right)-\mu T \\ 0\end{array}\right)$, and $G(X, Z)=P Z-\widehat{G}(X, Z)$ where

$$
\begin{aligned}
P & =\left(\begin{array}{cc}
\alpha T_{\max }(1-\vartheta)-\kappa & \beta T_{\max }(1-\rho) \\
\omega \kappa & -c
\end{array}\right) \\
\widehat{G}(X, Z) & =\left(\begin{array}{l}
\widehat{G_{1}}(X, Z) \\
\widehat{G_{2}}(X, Z)
\end{array}\right)=\left(\begin{array}{l}
0 \\
0
\end{array}\right)
\end{aligned}
$$

From (16) $\widehat{G_{1}}(X, Z)=\widehat{G_{2}}(X, Z)=0$ this implies that $\widehat{G}(X, Z) \geq 0$. Therefore, $E_{0}$ is globally asymptotically stable when $R_{0}<1$.

This means that, at any perturbation of the equilibrium point by the introduction of free virus particles, the model solutions will always converge to the IFE, whenever $R_{0}<1$.

\subsection{Existence of the Endemic Equilibrium (EE)}

Theorem 3. A positive endemic equilibrium EE exists provided $R_{0}>1$. 
Proof. The endemic equilibrium $E E=\left(T_{e}, T_{e}^{\star}, V_{e}\right)$ satisfies

$$
\begin{aligned}
& r T_{e}\left(1-\frac{T_{e}}{T_{\max }}\right)-(1-\rho) \beta V_{e} T_{e}-\alpha(1-\vartheta) T_{e} T_{e}^{\star} \\
& \quad-\mu T_{e}=0 \\
& (1-\rho) \beta V_{e} T_{e}+\alpha(1-\vartheta) T_{e} T_{e}^{\star}-\kappa T_{e}^{\star}=0 \\
& \omega \kappa T_{e}^{\star}-c V_{e}=0
\end{aligned}
$$

From (19) we have

$$
V_{e}=\frac{\omega \kappa T_{e}^{\star}}{c}
$$

Substituting (20) in (18) we get

$$
\begin{aligned}
T_{e} & =\frac{1}{(1-\rho)(\beta \omega / c)+(1-\vartheta)(\alpha / \kappa)} \\
& =\frac{T_{\max }(1-\mu / r)}{R_{0}}
\end{aligned}
$$

Substituting $V_{e}$ and $T_{e}$ in (17) we obtain

$$
T_{e}^{\star}=\frac{T_{\max }(1-\mu / r)\left(R_{0}-1\right)(r-\mu)}{\kappa R_{0}^{2}}
$$

The endemic equilibrium (EE) is given as

$$
E E=\left(\frac{T_{\max }(1-\mu / r)}{R_{0}}, \frac{T_{e}\left(R_{0}-1\right)(r-\mu)}{\kappa R_{0}}, \frac{\omega \kappa T_{e}^{\star}}{c}\right)
$$

Clearly $T_{e}^{\star}>0$ if and only if $R_{0}>1$.

\subsection{Local Stability Analysis of the Endemic Equilibrium}

Theorem 4. The endemic equilibrium $E E=\left(T_{e}, T_{e}^{\star}, V_{e}\right)$ is locally asymptotically stable whenever $R_{0}>1$.

Proof. The Jacobian matrix of model (1) at $E E$ is as follows:

$$
J(E E)=\left(\begin{array}{ccc}
\frac{(r-2)(r-\mu)}{r R_{0}} & \frac{-\alpha(1-9) T_{\max }(1-\mu / r)}{R_{0}} & \frac{-\beta(1-\rho) T_{\max }(1-\mu / r)}{R_{0}} \\
\frac{\left(R_{0}-1\right)(r-\mu)}{R_{0}} & \frac{\alpha(1-\vartheta) T_{\max }(1-\mu / r)}{R_{0}}-\kappa & \frac{(1-\rho) \beta T_{\max }(1-\mu / r)}{R_{0}} \\
0 & \omega \kappa & -c
\end{array}\right)
$$

The characteristic equation of (24) is in the form

$$
\lambda^{3}+a_{0} \lambda^{2}+a_{1} \lambda+a_{2}=0
$$

where

$$
\begin{aligned}
a_{0}= & \kappa+c-\frac{\alpha(1-\vartheta) T_{\max }(1-\mu / r)}{R_{0}} \\
& -\frac{(r-2)(r-\mu)}{r R_{0}} \\
a_{1} & =\left(\frac{(1-\vartheta) T_{\max }(1-\mu / r)}{R_{0}}\right) \\
& \cdot\left(\frac{(r-2)(r-\mu)}{r R_{0}}+\frac{\left(R_{0}-1\right)(r-\mu)}{R_{0}}\right) \\
& +\left(\frac{(r-2)(r-\mu)}{r R_{0}}\right)(c-\kappa)+c \kappa-1 \\
a_{2} & =\frac{\left(R_{0}-1\right)(r-\mu)}{R_{0}}+\frac{(r-2)(r-\mu)}{r R_{0}}(1-c \kappa)
\end{aligned}
$$

The number of possible negative real roots of (25) depends on the signs of $a_{0}, a_{1}$, and $a_{2}$. This can be established by applying Descartes Rule of Signs as used in [11].

$$
P(\lambda)=a_{1} \lambda^{2}+a_{2} \lambda+a_{3}
$$

According to this rule the number of negative real zeros of $P(\lambda)$ is either equal to the number of sign changes of $P(-\lambda)$ or less by an even number, as shown in Table 1 .

From Table 1 the maximum number of sign changes in $P(-\lambda)$ is 2 ; hence the characteristic polynomial (27) has two negative roots. Thus

$$
P(-\lambda)=-\lambda^{3}+a_{0} \lambda^{2}-a_{1} \lambda+a_{2}=0
$$

has negative real roots. Hence for $r>\mu$ and if cases 1 to 8 are satisfied then the endemic equilibrium EE is locally asymptotically stable.

Therefore if $R_{0}>1$ and given a small number of free virus particles, each virus, in the entire period of its infectivity, will produce on average more than one infected cell, implying viral persistence.

3.6. Global Stability Analysis of the Endemic Equilibrium. In this section we investigate the global stability of the endemic equilibrium using geometric approach, as developed by Li and Muldowney in [15]. For a brief description of this approach, see $[6,8]$. Consider the autonomous dynamical system

$$
y^{\prime}=f(x)
$$


TABLE 1: Roots of characteristic equation (27).

\begin{tabular}{lcccccc}
\hline cases & $a_{0}$ & $a_{1}$ & $a_{2}$ & $R_{0}>1$ & no. of sign changes & no. of real -ve roots \\
\hline 1 & - & + & - & $R_{0}>1$ & 2 & 2,0 \\
2 & - & - & - & $R_{0}>1$ & 0 & 0 \\
3 & + & + & - & $R_{0}>1$ & 1 & 0 \\
4 & + & - & - & $R_{0}>1$ & 1 & 0 \\
5 & - & + & + & $R_{0}>1$ & 1 & 0 \\
6 & - & - & + & $R_{0}>1$ & 1 & 0 \\
7 & + & + & + & $R_{0}>1$ & 0 & 0 \\
8 & + & - & + & $R_{0}>1$ & 2 & 2,0 \\
\hline
\end{tabular}

where $f: \Omega \longrightarrow \mathbb{R}^{n}, \Omega \subset \mathbb{R}^{n}$ is an open set and is simply connected and $y \in \Omega, y \longmapsto f(x) \in \mathbb{R}^{n}, f(x) \in C^{\prime}(\Omega)$. Let $y^{\star}$ be an equilibrium point, then $y^{\star}$ is said to be globally stable in $\Omega$ if it is locally stable in $\Omega$ and all trajectories in $\Omega$ converge to $y^{\star}$. In this method the equilibrium $y^{\star}$ is locally asymptotically stable provided the following conditions hold:

(i) (H1) $\Omega$ is simply connected

(ii) (H2) There exists a compact absorbing set $K \subset \Omega$

(iii) (H3) Equation (29) has a unique equilibrium $y^{\star}$ in $\Omega$

Let $P(y)$ be a $\left(\begin{array}{c}n \\ 2\end{array}\right) \times\left(\begin{array}{l}n \\ 2\end{array}\right)$ matrix-valued function that is $C^{\prime}$ on $\Omega$ and consider $B=P_{f} P^{-1}+P\left(\partial f^{2} / \partial y\right) P^{-1}$ where the matrix $P_{f}$ is $\left(\partial P_{i j}^{\star} / \partial y\right) f=d P_{i j} / d t$ and let the matrix $J^{(2)}$ be the second additive compound matrix of the Jacobian matrix $J$; that is, $J_{(y)}=\left(J_{i j}\right), J^{(2)}$ is a $\left(\begin{array}{l}n \\ 2\end{array}\right) \times\left(\begin{array}{l}n \\ 2\end{array}\right)$ matrix, and in our case $\mathrm{n}=3$; hence

$$
J^{(2)}=\left(\begin{array}{ccc}
J_{11}+J_{22} & J_{23} & -J_{13} \\
J_{32} & J_{11}+J_{33} & J_{12} \\
-J_{31} & J_{21} & J_{22}+J_{33}
\end{array}\right)
$$

Consider the Lonziskii measure $\mu$ of $B$ with respect to a vector norm $|\cdot|$ in $\mathbb{R}^{N}$ where $N=\left(\begin{array}{c}n \\ 2\end{array}\right)$

$$
\mu(B)=\lim _{t \rightarrow 0^{+}} \frac{\|I+h B\|-1}{h}
$$

It is proved in [15] that if (H1), (H2), and (H3) hold and condition

$$
\bar{q}=\limsup _{t \rightarrow \infty} \sup _{y_{0} \in K} \frac{1}{t} \int_{0}^{t} \mu\left(B\left(x\left(s, x_{0}\right)\right)\right) d s<0
$$

is satisfied, then the unique equilibrium $y^{\star}$ is globally asymptotically stable

Lemma 5. Assume that conditions (H1), (H2), and (H3) hold, then $y^{\star}$ is globally asymptotically stable in $\Omega$ provided that a function $P(x)$ and a Lonziskii measure [16] $\mu$ exist such that condition (32) is satisfied.

Theorem 6. The endemic equilibrium EE is globally asymptotically stable in $\Omega$ if $R_{0}>1$.

Proof. Consider the Jacobian of model (1)

$$
J=\left(\begin{array}{ccc}
r-\frac{2 r T}{T_{\max }}-(1-\rho) \beta V-(1-\vartheta) \alpha T^{\star}-\mu & -(1-\vartheta) \alpha T & -(1-\rho) \beta T \\
(1-\rho) \beta V+(1-9) \alpha T^{\star} & (1-\vartheta) \alpha T-\kappa & (1-\rho) \beta T \\
0 & \omega \kappa & -c
\end{array}\right)
$$

and the second compound additive matrix of (33) is given as

$$
J^{(2)}=\left(\begin{array}{ccc}
a+b-\kappa & d & d \\
\omega \kappa & a-c & -b \\
0 & e & b-\kappa-c
\end{array}\right)
$$

where

$$
\begin{aligned}
a & =r-\frac{2 r T}{T_{\max }}-(1-\rho) \beta V-\alpha(1-\vartheta) T^{\star}-\mu \\
b & =\alpha T(1-\vartheta) \\
d & =(1-\rho) \beta T \\
e & =(1-\rho) \beta V+\alpha T^{\star}(1-\vartheta)
\end{aligned}
$$

We define an auxiliary matrix function $Q$ on $\Omega$ as

$$
Q=\operatorname{diag}\left(\frac{1}{T^{\star}}, \frac{1}{V}, \frac{1}{V}\right)
$$

setting $T^{\star}, V>0$ everywhere in $\Omega$, and $Q$ is smooth and nonsingular. $Q_{f}$ and $Q_{f} Q^{-1}$ are given as

$$
\begin{aligned}
Q_{f} & =\operatorname{diag}\left(-\frac{T^{\star \star}}{\left(T^{\star}\right)^{2}},-\frac{\dot{V}}{V^{2}},-\frac{\dot{V}}{V^{2}}\right) \\
Q_{f} Q^{-1} & =\operatorname{diag}\left(-\frac{T^{\star}}{T^{\star}},-\frac{\dot{V}}{V},-\frac{\dot{V}}{V}\right)
\end{aligned}
$$


where $T^{\star}=d T^{\star} / d t$ and $\dot{V}=d V / d t$. Matrix $Q J^{(2)} Q^{-1}$ is given as

$$
Q J^{(2)} Q^{-1}=\left(\begin{array}{ccc}
a+b-\kappa & \frac{d V}{T^{\star}} & \frac{d V}{T^{\star}} \\
\frac{\omega \kappa T^{\star}}{V} & a-c & -b \\
0 & e & b-\kappa-c
\end{array}\right)
$$

Thus the matrix $M=Q_{f} Q^{-1}+Q J^{(2)} Q^{-1}$ as defined in (4.4) of [15] can be written in block form as

$$
M=\left(\begin{array}{ll}
m_{11} & m_{12} \\
m_{21} & m_{22}
\end{array}\right)
$$

where $m_{11}=a+b-\kappa-\dot{T} \star / T^{\star}, m_{12}=d V / T^{\star}, d V / T^{\star}$, $m_{21}=\left(\begin{array}{c}\omega \kappa T^{\star} / V \\ 0\end{array}\right)$, and $m_{22}=\left(\begin{array}{cc}a-c-\dot{V} / V & -b \\ e & b-c-\kappa-\dot{V} / V\end{array}\right)$. Let the

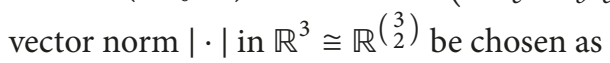

$$
|(u, v, w)|=\sup \{|u|,|v|+|w|\}
$$

The Lozinskii measure $\mu(M)$ with respect to $|\cdot|$ can be estimated as follows:

$$
\mu(M) \leq \sup \left\{g_{1}, g_{2}\right\}
$$

where

$$
\begin{aligned}
& g_{1}=\mu_{1}\left(m_{11}\right)+\left\|m_{12}\right\| \\
& g_{2}=\mu_{1}\left(m_{22}\right)+\left\|m_{21}\right\|
\end{aligned}
$$

$\left\|m_{12}\right\|$ and $\left\|m_{21}\right\|$ are operator norms associated with the linear mappings $m_{12}: \mathbb{R}^{2} \longrightarrow \mathbb{R}$ and $m_{12}: \mathbb{R} \longrightarrow \mathbb{R}^{2}$, respectively, where $\mathbb{R}$ is endowed with the $\ell_{1}$ vector norm in both cases. Specifically $\mu_{1}\left(m_{11}\right)=a+b-\kappa-i \star / T^{\star}$, $\left\|m_{12}\right\|=d V / T^{\star},\left\|m_{21}\right\|=\sup \left\{\omega \kappa T^{\star} / V, 0\right\}$, and $\mu_{1}\left(m_{22}\right)=$ $-c-\dot{V} / V+\sup \{a+e,-\kappa\}$. From model (1) we find

$$
\begin{aligned}
\frac{T^{\star}}{T^{\star}} & =(1-\rho) \beta V T+(1-\vartheta) \alpha T-\kappa \\
\frac{\dot{V}}{V} & =\frac{\omega \kappa T^{\star}}{V}-c
\end{aligned}
$$

and recalling expressions for $a, b, d$, and $e$ we obtain

$$
\begin{aligned}
& g_{1}=r-\frac{2 r T}{T_{\max }}-(1-\rho) \beta V-(1-\vartheta) \alpha T^{\star}-\mu \\
& g_{2}=\sup \left\{r-\frac{2 r T}{T_{\max }}-\mu,-\kappa\right\}
\end{aligned}
$$

Since $r-2 r T / T_{\text {max }}-(1-\rho) \beta V-(1-\vartheta) \alpha T^{\star}-\mu \leq \sup \{r-$ $\left.2 r T / T_{\max }-\mu,-\kappa\right\}$ we find that $g_{1} \leq g_{2}$, then (42) implies that $\mu(M) \leq g_{2}$; thus

$$
\begin{aligned}
\mu(M) \leq & r-\frac{2 r T}{T_{\max }}-\mu \\
& +\sup \left\{-(1-\rho) \beta V-(1-\vartheta) \alpha T^{\star}, 0\right\} \\
\leq & r-\frac{2 r T}{T_{\max }}-\mu
\end{aligned}
$$

Since $T_{\text {max }}$ is the limiting value of $T(t)$, then this implies that $\lim \sup _{t \rightarrow \infty} T(t) \leq T_{\text {max }}$; therefore expression (49) reduces to

$$
\mu(M) \leq r-2 r-\mu
$$

Integrating (50) we obtain

$$
\frac{1}{t} \int_{0}^{t} \mu(M) d s \leq \frac{1}{t} \int_{0}^{t}(r-2 r-\mu) d s<0
$$

Hence the endemic equilibrium (EE) is globally asymptotically stable whenever $R_{0}>1$.

This implies that, regardless of any starting solution, the solution of the model will converge to EE whenever $R_{0}>$ 1. Immunologically, it means that, at any perturbation of the equilibrium point as a result of the introduction of the free virus particles, the model solutions will converge to the endemic state.

\section{Numerical Simulation of the Model}

In this section we perform a numerical simulation of model (1) using MATLAB with the parameter values given in Table 1. The primary purpose of the numerical simulation is to analyze the change in state of virus progression with time and also to outline the impact of the variation of treatment efficacy on the transmission dynamics of HIV. This is achieved by varying the parameter values $\rho$ and $\vartheta$ while keeping the other parameters constant.

4.1. Effect of Variations in RTI and PI Treatment Efficacy on the Asymptotic Behaviour of the Equilibrium Points. The effect of variations in combined treatment efficacy on the stability of equilibria is investigated using the parameter values given in Table 2; this is achieved by choosing four different initial conditions
(i) ICl: $T_{1}(t)=1000, T_{1}^{\star}(t)=0, V_{1}(t)=10^{-3}$
(ii) IC2: $T_{2}(t)=800, T_{2}^{\star}(t)=10, V_{2}(t)=0.001$
(iii) IC3: $T_{3}(t)=900, T_{3}^{\star}(t)=5, V_{3}(t)=0.01$
(iv) IC4: $T_{4}(t)=1000, T_{4}^{\star}(t)=1, V_{4}(t)=0.1$

and varying parameters $\rho$ and $\vartheta$ while keeping other parameters constant. From Theorems 2 and 6, the corresponding stabilities of model (1) are as follows:

(1) If $R_{0}<1$, the IFE is globally asymptotically stable.

(2) If $R_{0}>1$, the EE is globally asymptotically stable.

(i) $\rho=0.1$ and $\vartheta=0.1$. For this set of parameters we obtain $R_{0}=4.545>1$. By Theorem 6 the endemic equilibrium is globally asymptotically stable and the states of the system converge to $E E=$ $(110.011,3.575,357.53)$, for the four initial conditions IC1-IC4, implying that the virus persists in the host. This shows that the low treatment efficacy of 0.1 cannot effectively combat the virus. This is illustrated by Figures 1, 2, 3, and 4 . 
TABLE 2: Parameters values for model (1).

\begin{tabular}{|c|c|c|c|}
\hline Parameters & units & Description & source \\
\hline$T_{\max }$ & 1500 cells $\mathrm{mm}^{-3}$ & Maximum CD4+ cell population level & [9] \\
\hline$r$ & 0.03 cells $d a y^{-1}$ & $\begin{array}{l}\text { Production rate of } \\
\text { uninfected T cells }\end{array}$ & [9] \\
\hline$\mu$ & 0.02 cells $d a y^{-1}$ & $\begin{array}{l}\text { Natural death rate of } \\
\text { uninfected T cells }\end{array}$ & [9] \\
\hline$\kappa$ & 0.24 cells $d a y^{-1}$ & $\begin{array}{l}\text { Death rate of actively } \\
\text { infected T cells }\end{array}$ & [9] \\
\hline$c$ & 2.4 day $^{-1}$ & Shedding rate of virions & [9] \\
\hline$\beta$ & $2.4 \times 10^{-5} \mathrm{~mm}^{-3}$ & $\begin{array}{l}\text { Viral infection rate } \\
\text { by free virions }\end{array}$ & [9] \\
\hline$\alpha$ & $2.4 \times 10^{-5} \mathrm{~mm}^{-3}$ & cellular infection rate & [9] \\
\hline$\omega$ & varies: $\omega \geq 0$ & $\begin{array}{l}\text { Burst rate of actively } \\
\text { infected } T \text { cells }\end{array}$ & - \\
\hline$\rho$ & varies: $0<\rho<1$ & Efficacy of RT Inhibitor & - \\
\hline$\vartheta$ & varies: $0<9<1$ & efficacy of Protease Inhibitor & - \\
\hline
\end{tabular}

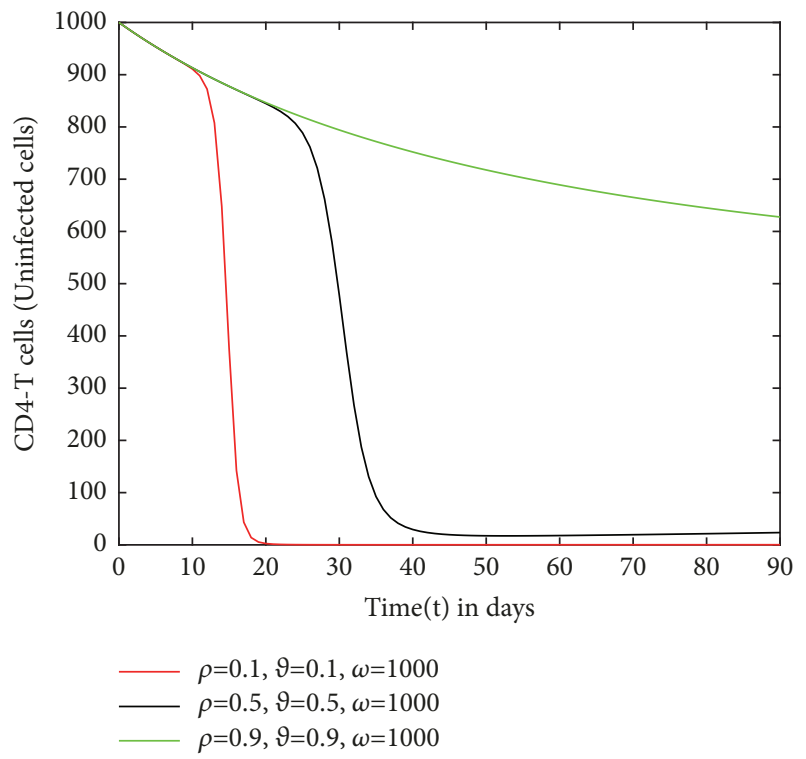

(a)

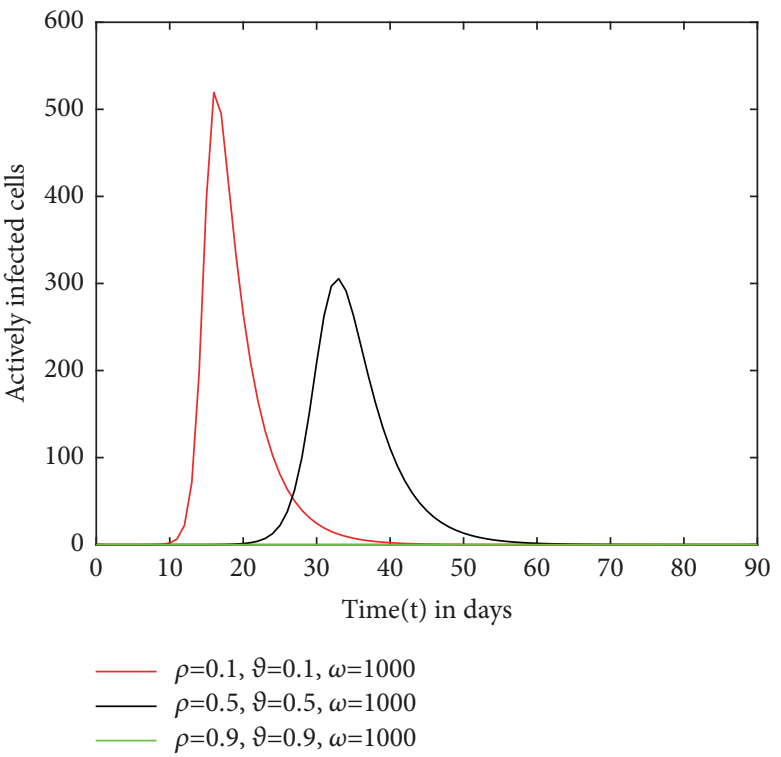

(b)

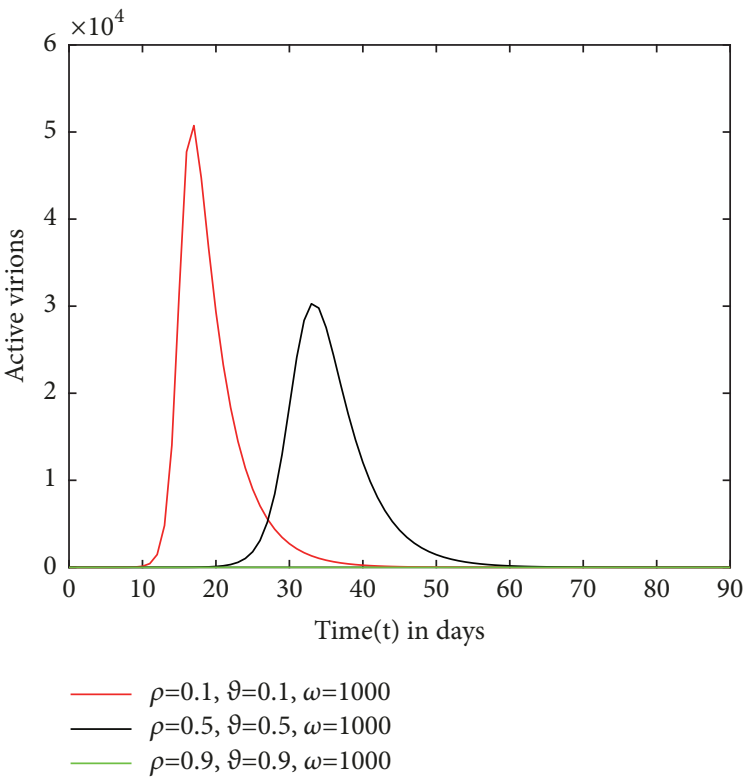

(c)

FiguRE 1: Effect of treatment efficacy on $T(t), T^{\star}(t)$, and $V(t)$. 


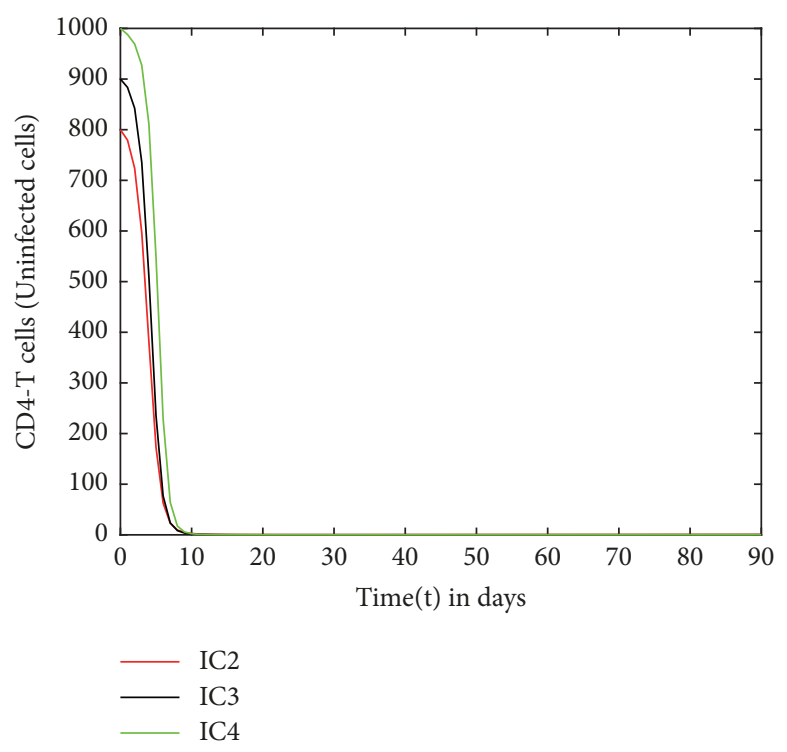

(a)

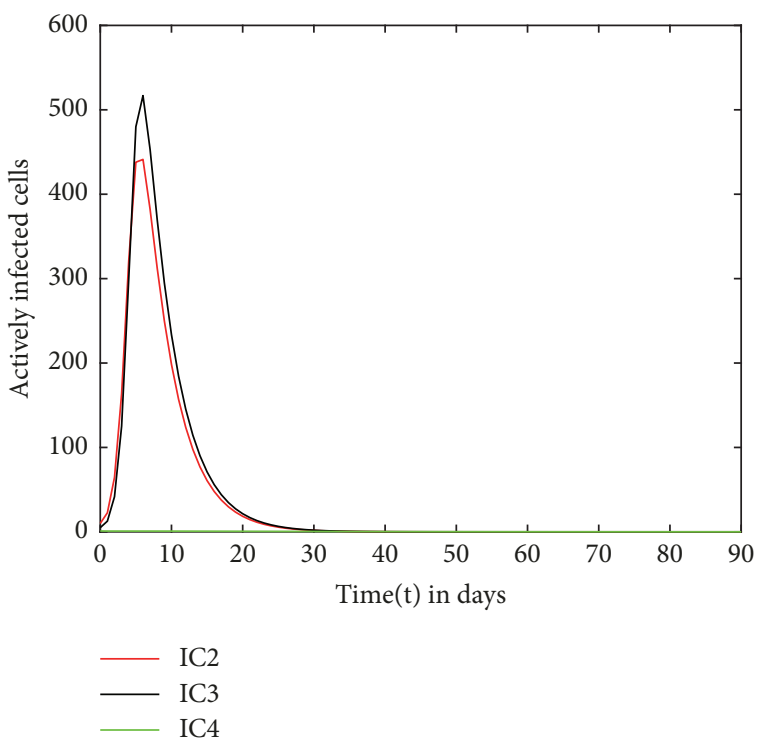

(b)

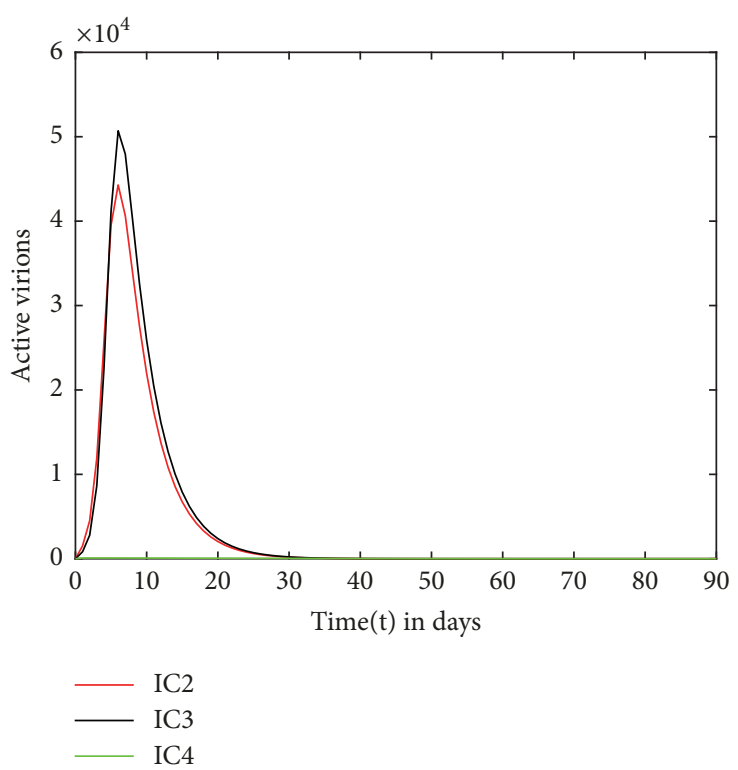

(c)

FIgURE 2: (a)-(c) The graph trajectory of $T(t), T^{\star}(t)$, and $V(t)$ for $\rho=0.1$ and $\vartheta=0.1$ with the initial conditions IC2-IC4. $R_{0}=4.545$ and $E E=(110.011,3.575,357.53)$ is GAS.

(ii) $\rho=0.5$ and $\mathcal{\vartheta}=0.5$. This set of parameters yields $R_{0}=2.525>1$ and the system state approach $E E=$ $(198.02,4.98,498.31)$ for all the four initial conditions IC1-IC4. This means that EE exists and is globally asymptotically stable according to Theorem 6 . Again despite the increase in treatment efficacy the virus persists in the host. Figures 1, 2, 3, and 4 illustrate this scenario.

(iii) $\rho=0.82$ and $\vartheta=0.9$. This treatment efficacy yields $R_{0}=0.101<1$ and by Theorem $2 E_{0}=(1500,0,0)$ exists and is GAS for all initial values IC1-IC4, as can be observed from Figures 1, 2, 3, and 4 implying that the high treatment efficacy of over 0.82 helps in stifling viral replication within host.

4.2. Effect of Variations in RTI and PI Treatment Efficacy on the CD4+ T Cell Population and Viral Load. From Figures 1(a), $1(\mathrm{~b})$, and $1(\mathrm{c})$, it is observed that variations in the treatment efficacy have significant effect on the number of CD4+ T cells. For instance, with a treatment efficacy of 0.1 at the onset of $\mathrm{HIV}$ infection, the CD4+ T cells undergo a sharp decline, while treatment efficacy of 0.5 only slows down CD4+ T cell depletion and takes longer (approximately 40 days) to reduce to zero. However with a drug efficacy of 0.9 the number of 


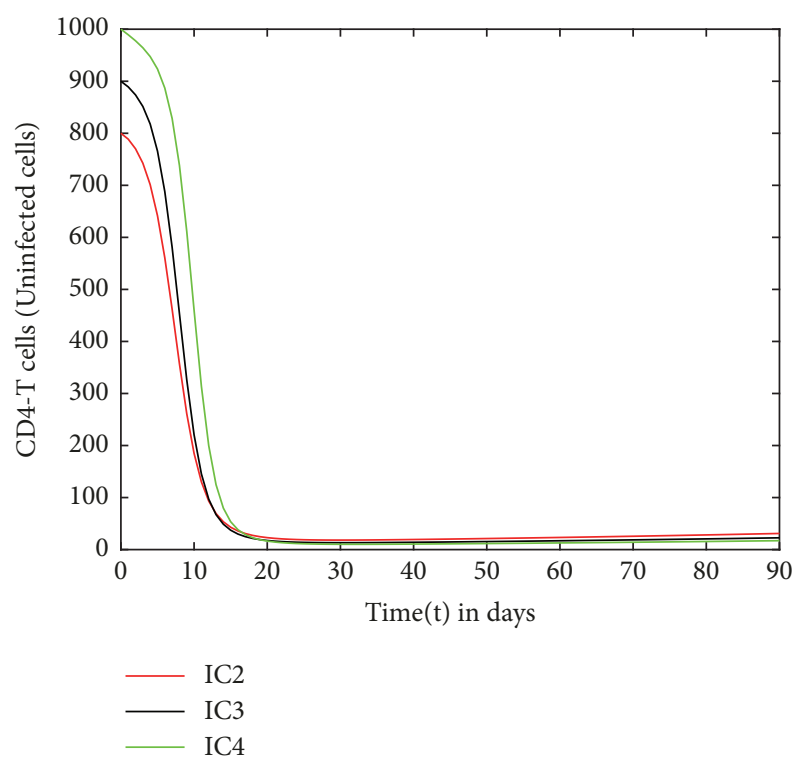

(a)

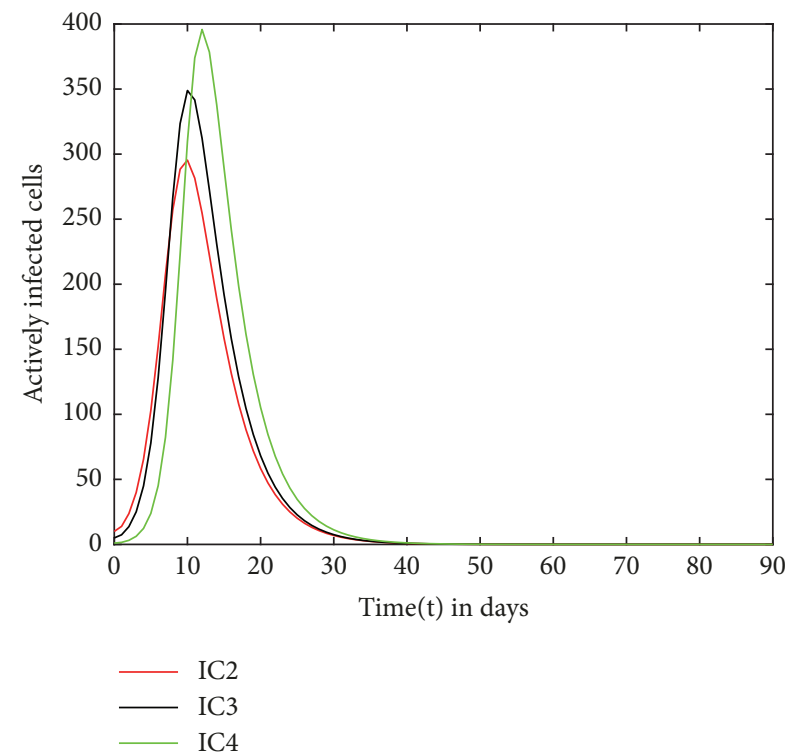

(b)

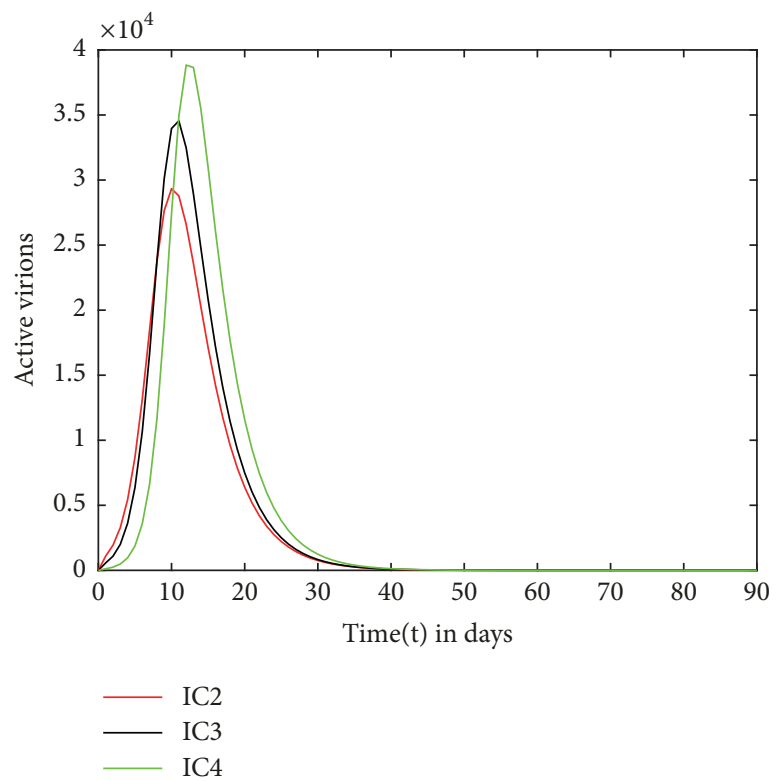

(c)

FIgURE 3: (a)-(c) The graph trajectory of $T(t), T^{\star}(t)$, and $V(t)$ for $\rho=0.5$ and $9=0.5$ with the initial conditions IC2-IC4. $R_{0}=2.525>1$ and $E E=(198.02,4.98,498.31)$ is GAS.

CD4+ T cells is kept above $700 \mathrm{~mm}^{-3}$. This demonstrates that treatment efficacy is directly proportional to the number of CD4+ T cells.

Figures 1(b) and 1(c) illustrate the effect of treatment efficacy on the number of actively infected cells and free virions, respectively. It is clear that from the time of infection if treatment efficacy is kept at a minimum of 0.1 , then both actively infected cells and free virions replicate rapidly attaining a primary peak of 519 infected cells within the first 17 days, while the virions reach a peak of $5.074 \times 10^{4}$ within the same period of time. With an increase in treatment efficacy both infected cells and viral replication rates are significantly reduced; for instance, at 0.9 efficacy level the number of virus and infected cells approach zero within the first 90 days.

\section{Conclusion}

In this paper, a mathematical model for within host HIV infection with virus-to-cell and cell-to-cell treatment has been formulated and analyzed. Target cell production has been modelled by a logistic incidence rate. The global dynamics have been shown to be dependent on $R_{0}$, in which for 


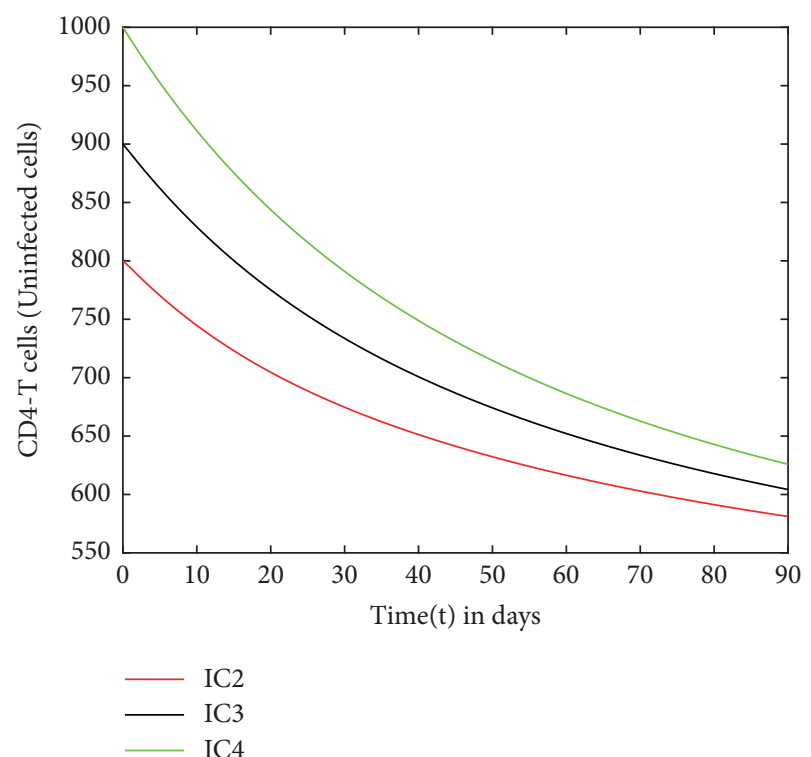

(a)

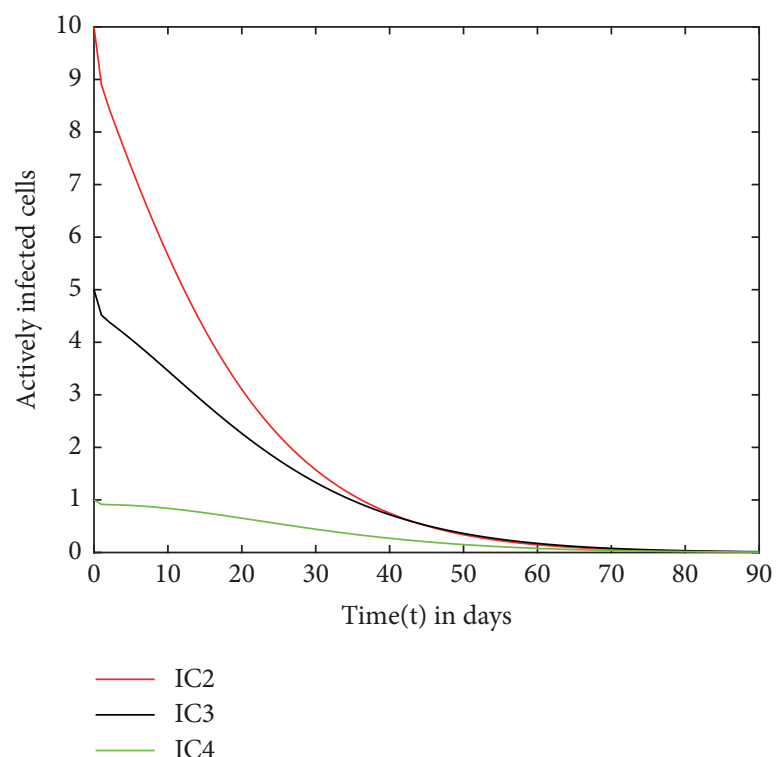

(b)

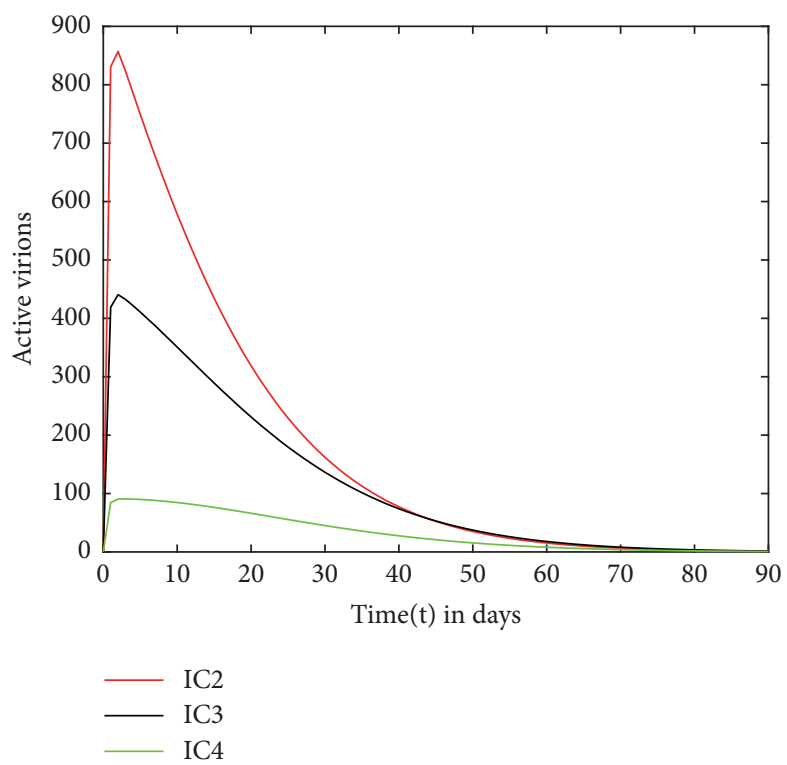

(c)

Figure 4: (a)-(c) The graph trajectory of $T(t), T^{\star}(t)$, and $V(t)$ for $\rho=0.9$ and $\vartheta=0.9$ with the initial conditions IC2-IC4. $R_{0}=0.101<1$ and $E_{0}=(1500,0,0)$ is globally asymptotically stable.

values of $R_{0}<1$ the infection free equilibrium is globally asymptotically stable, and for $R_{0}>1$ the endemic equilibrium is globally asymptotically stable. The global asymptotic stability of IFE and EE has been proved using the method by Castillo-Chavez and the geometric approach, respectively. Numerical simulations have shown that combined treatment has significant effect on the dynamics of HIV infection, in which high levels of treatment efficacy for both RTI and PI treatment was observed to stifle viral replication. This observation is in agreement with the findings of [9] which showed that if $R_{0}>1$ before treatment then the virus will increase as would be the number of infected cells, but after treatment if $R_{0}<1$, then $T^{\star}$ and $V$ would both decline.

\section{Data Availability}

Previously reported data were used to support this study and are available at [9]. These prior studies (and datasets) are cited at relevant places within the text as references.

\section{Conflicts of Interest}

The authors declare that there are no conflicts of interest regarding the publication of this paper. 


\section{References}

[1] M. A. Nowak and R. M. May, Virus Dynamics: Mathematics Principles of Immunology and Virology, Oxford University Press, London, UK, 2000.

[2] S.-S. Chen, C.-Y. Cheng, and Y. Takeuchi, "Stability analysis in delayed within-host viral dynamics with both viral and cellular infections," Journal of Mathematical Analysis and Applications, vol. 442, no. 2, pp. 642-672, 2016.

[3] A. M. Elaiw and N. A. Almuallem, "Global dynamics of delaydistributed HIV infection models with differential drug efficacy in cocirculating target cells," Mathematical Methods in the Applied Sciences, vol. 39, no. 1, pp. 4-31, 2016.

[4] A. M. Elaiw and A. A. Raezah, "Stability of general virus dynamics models with both cellular and viral infections and delays," Mathematical Methods in the Applied Sciences, vol. 40, no. 16, pp. 5863-5880, 2017.

[5] Y. Yang, L. Zou, and S. Ruan, "Global dynamics of a delayed within-host viral infection model with both virus-to-cell and cell-to-cell transmissions," Mathematical Biosciences, vol. 270, no. part B, pp. 183-191, 2015.

[6] H. Pourbashash, S. S. Pilyugin, P. De Leenheer, and C. McCluskey, "Global analysis of within host virus models with cell-to-cell viral transmission," Discrete and Continuous Dynamical Systems - Series B, vol. 19, no. 10, pp. 3341-3357, 2014.

[7] X. Zhang and X.-N. Liu, "Backward bifurcation of an epidemic model with saturated treatment function," Journal of Mathematical Analysis and Applications, vol. 348, no. 1, pp. 433-443, 2008.

[8] J. Zhang, J. Jia, and X. Song, "Analysis of an SEIR epidemic model with saturated incidence and saturated treatment function," The Scientific World Journal, vol. 2014, 2014.

[9] A. S. Perelson, D. E. Kirschner, and R. D. Boer, "Dynamics of HIV infection of CD $4^{+}$T cells," Mathematical Biosciences, vol. 114, no. 1, pp. 81-125, 1993.

[10] D. E. Kirschner and G. F. Webb, "A Mathematical Model of Combined Drug Therapy of HIV Infection," Journal of Theoretical Medicine, vol. 1, no. 1, pp. 25-34, 1997.

[11] F. K. Tireito, G. O. Lawi, and C. O. Okaka, "Mathematical analysis of HIV/AIDS prophylaxis treatment model," Applied Mathematical Sciences, vol. 12, no. 18, pp. 893-902, 2018.

[12] S. Pankavich, "The effects of latent infection on the dynamics of HIV," Differential Equations and Dynamical Systems, vol. 24, no. 3, pp. 281-303, 2016.

[13] C. Castillo-Chavez, Z. Feng, and W. Huang, "On the computation of R0 and its role on global stability," in Mathematical Approaches for Emerging and Reemerging Infectious Diseases: An Introduction, C. Castillo-Chavez, S. Blower, P. van den Driessche, D. Kirschner, and A.-A. Yakubu, Eds., vol. 125, pp. 229-250, Springer, New York, NY, USA, 2002.

[14] Van Den Driessche and J. Wu, Mathematical Epidemiology, Springer-Varleg, Berlin Heidelberg, Germany, 2008.

[15] M. Y. Li and J. S. Muldowney, "A geometric approach to globalstability problems," SIAM Journal on Mathematical Analysis, vol. 27, no. 4, pp. 1070-1083, 1996.

[16] W. A. Coppel, Stability and Asymptotic Behavior of Differential Equations, D. C. Heath, Boston, Mass, USA, 1965. 


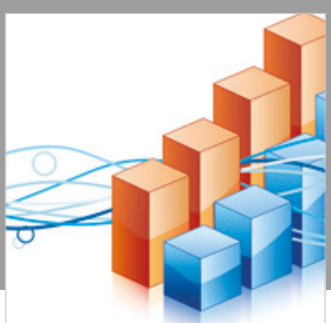

Advances in

Operations Research

\section{-n-m}
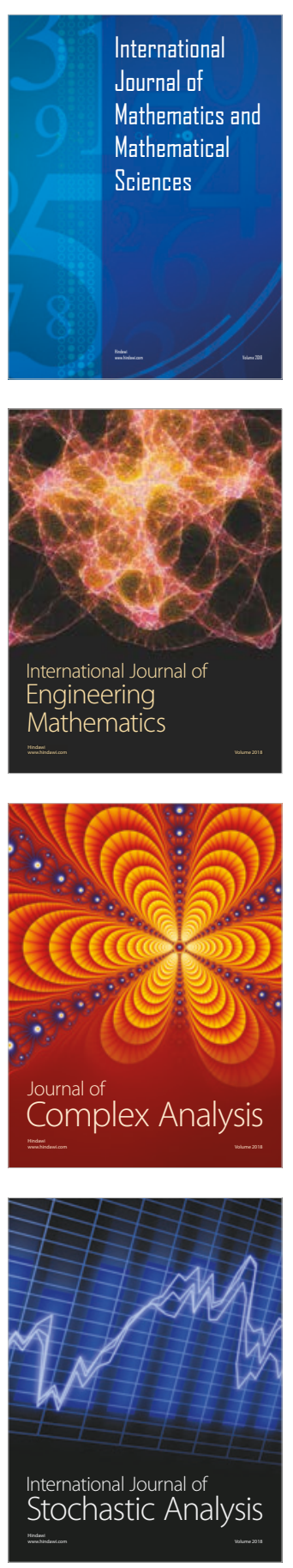
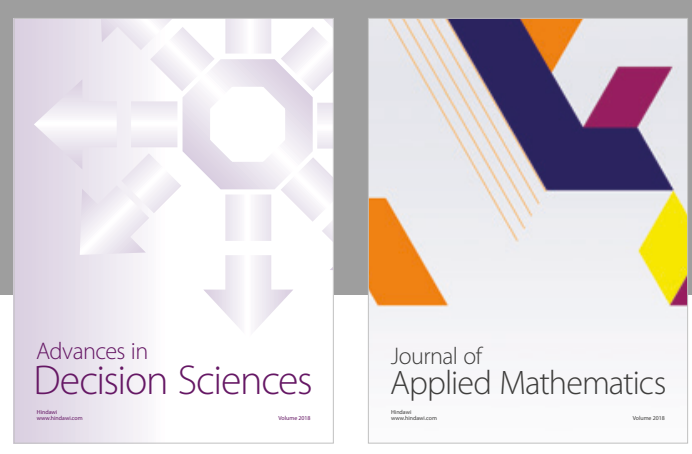

Journal of

Applied Mathematics
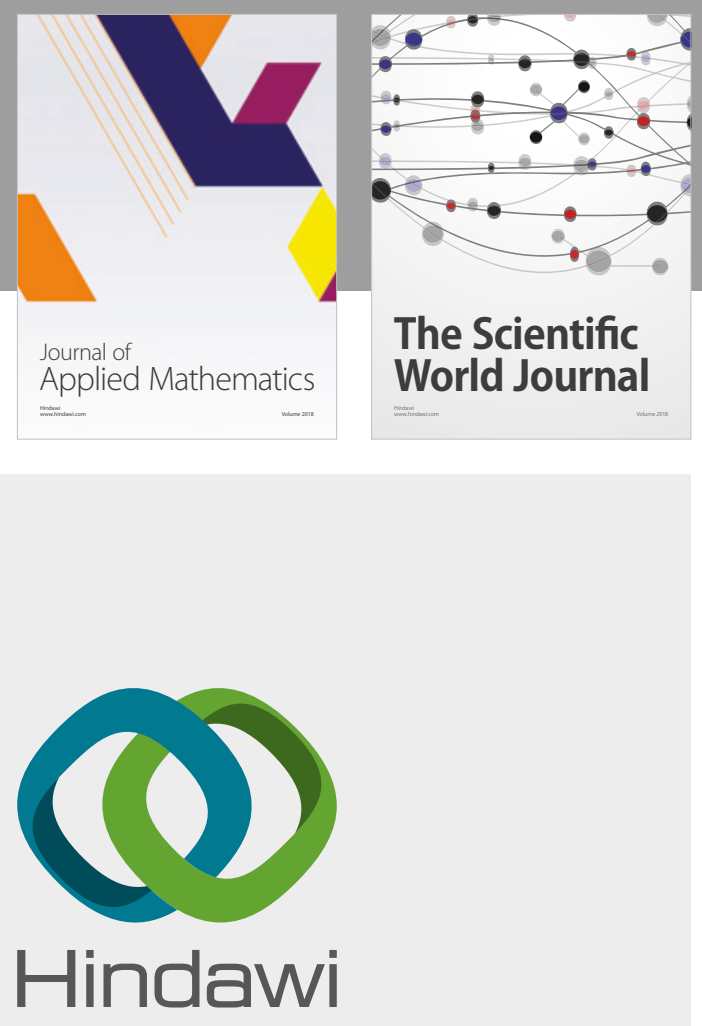

Submit your manuscripts at

www.hindawi.com

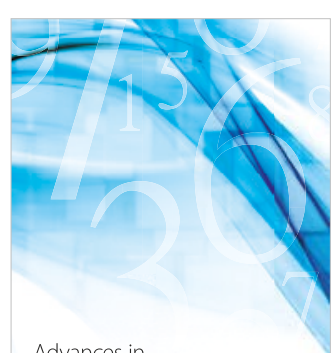

Advances in
Numerical Analysis
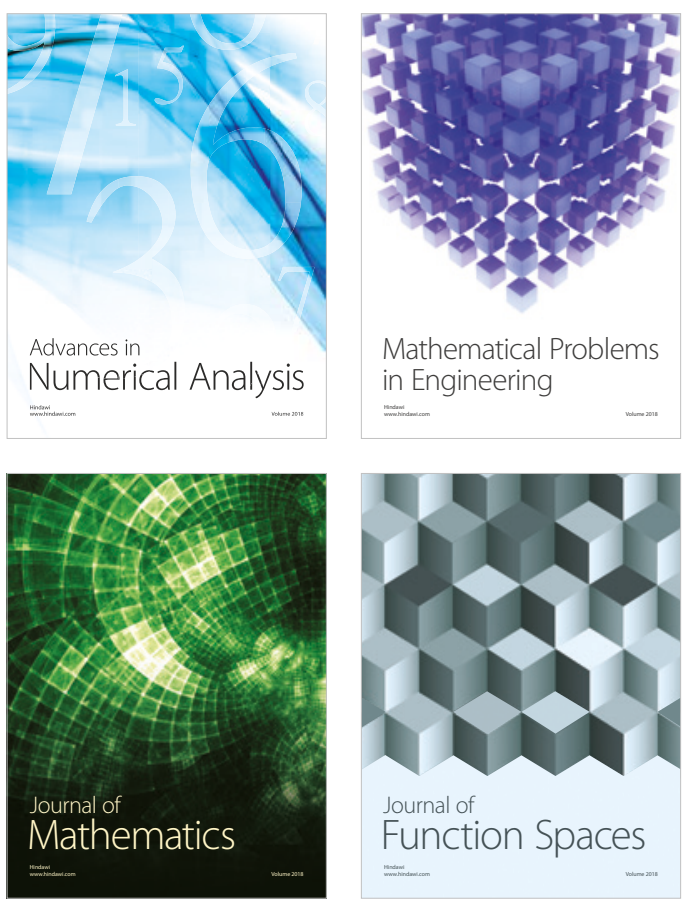

Mathematical Problems in Engineering

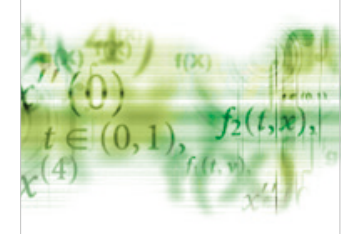

International Journal of

Differential Equations

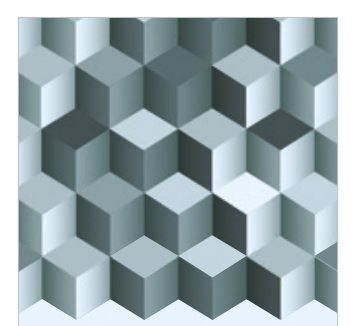

Journal of

Function Spaces

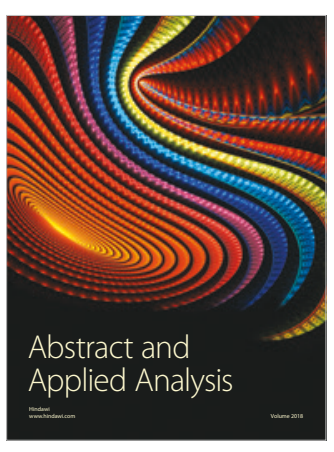

The Scientific

World Journal

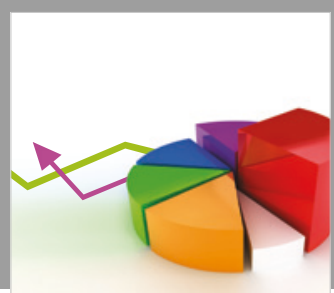

Journal of

Probability and Statistics
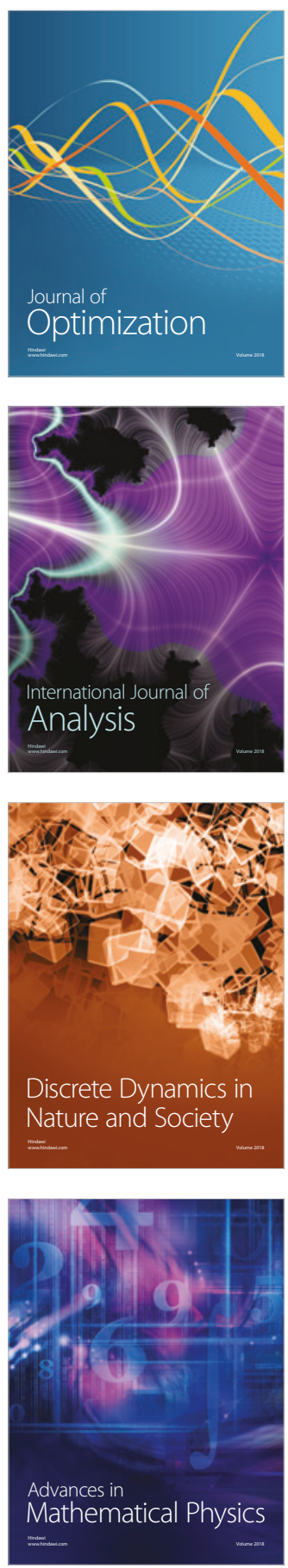